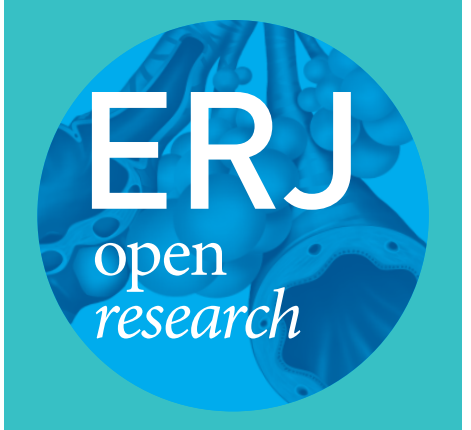

\title{
Identification of target zones for lung volume reduction surgery using three- dimensional computed tomography rendering
}

\author{
Claudio Caviezel ${ }^{1}$, Tamara Froehlich ${ }^{1}$, Didier Schneiter ${ }^{1}$, Urs Muehlematter ${ }^{2}$, \\ Thomas Frauenfelder ${ }^{2}$, Laura-Chiara Guglielmetti ${ }^{1}$, Isabelle Opitz ${ }^{1}$ and \\ Walter Weder ${ }^{1}$
}

Affiliations: ${ }^{1}$ Dept of Thoracic Surgery, University Hospital Zurich, Zurich, Switzerland. ${ }^{2}$ Institute for Interventional and Diagnostic Radiology, University Hospital Zurich, Zurich, Switzerland.

Correspondence: Walter Weder, Thoraxchirurgie Bethanien, Toblerstrasse 61, 8044 Zurich, Switzerland. E-mail: w.wederathorax-zuerich.ch

\section{ABSTRACT}

Background: The key issues for performing lung volume reduction surgery (LVRS) is the identification of the target zones. Recently introduced three-dimensional computed tomography rendering methods are used to identify the morphological distribution and its severity of lung emphysema by densitometry. We demonstrate a new software for emphysema imaging and show the pre- and post-operative results in patients undergoing LVRS planned based on this new technology.

Methods: A real-time three-dimensional image analysis software system was used pre- and 3 months postoperatively in five patients with heterogeneous emphysema and a single patient with homogeneous morphology scheduled for LVRS. Focus was on low attenuation areas with $<950 \mathrm{HU}$, distribution on both lungs and the value of the three-dimensional images for planning surgery. Functional outcome was assessed by pulmonary function tests after 3 months.

Results: Five patients underwent bilateral LVRS and one patient had unilateral LVRS. All patients showed a median increase in forced expiratory volume in $1 \mathrm{~s}$ of $70 \%$ (range 30-120\%), compared with baseline values. Hyperinflation (expressed as residual volume/total lung capacity ratio) was reduced by $30 \%$ (range 5-32\%). In the patients with heterogeneous emphysema, the pre- and post-operative computed tomography scans and the densitometries showed a decrease in low attenuation areas by $23 \%$ (right side) and by $17 \%$ (left side), respectively.

Conclusion: We demonstrate three-dimensional computed tomography-rendered images for planning personalised remodelling of hyperinflated lungs using LVRS. This user-friendly software has the potential to assist surgeons and interventional pulmonologists to select patients and to visualise target areas in LVRS procedures.

@ERSpublications

New, user-friendly software with 3D CT-rendered images can be used for planning personalised remodelling of hyperinflated lungs using LVRS https://bit.ly/3fbICn2

Cite this article as: Caviezel C, Froehlich T, Schneiter D, et al. Identification of target zones for lung volume reduction surgery using three-dimensional computed tomography rendering. ERJ Open Res 2020; 6: 00305-2020 [https://doi.org/10.1183/23120541.00305-2020].

Received: 25 May 2020 | Accepted after revision: 4 June 2020

Copyright $\odot$ ERS 2020. This article is open access and distributed under the terms of the Creative Commons Attribution Non-Commercial Licence 4.0. 


\section{Introduction}

Lung volume reduction surgery (LVRS) is intended to remodel hyperinflated lungs to normal size by resecting the most functionless parts of the emphysematous lung in symptomatic patients with COPD $[1,2]$. Volume reduction results primarily in improved respiratory mechanics and hence to a decrease in dyspnoea, an improvement of lung function and an increase of quality of life $[3,4]$.

Patient selection is of great importance and based on lung function values, particularly the severity of hyperinflation on one hand and on emphysema morphology assessed by computed tomography (CT) scans and perfusion scintigraphy on the other hand $[5,6]$.

The key issues in LVRS are: 1) identification of the optimal resection areas; 2) defining the volume to be resected, aiming to reduce hyperinflation to the predicted total lung capacity (TLC); and 3) the translation of both into an operation plan. The surgeon identifies these areas currently based on CT images by combining the radiological findings from CT scans with perfusion scintigraphy and modifies it by the intra-operative macroscopic appearance. Purely visual scoring on CT images, regarding the quantification of emphysema severity, was shown to have high interobserver variability and is largely dependent on the experience of the reader [6]. A so-called semi-quantitative method using the Goddard score showed a higher interobserver agreement, but the assessment needs to be performed by experienced and specially trained radiologists $[7,8]$. Nevertheless, in the National Emphysema Treatment Trial, previously trained chest radiologists still showed significant inter- and intra-observer disagreement [6]. As described recently [9], colour-coded volume-rendered CT has a high accuracy and is less observer-dependent. This so-called three-dimensional (3D) CT densitometry might be useful to analyse emphysema distribution as well as to identify target areas for resection.

Pre-operative simulation for thoracic surgical procedures using CT-based 3D reconstruction has been recently introduced $[10,11]$. This software-based planning was primarily applied for anatomical sublobar resection in patients with small pulmonary nodules and for estimating the final lobar size in living lobar lung transplantation [12]. The software automatically creates colour-coded images of the lung parenchyma, allowing the characterisation of locoregional emphysema distribution and severity, the extension and distribution. The software can be applied on any CT scans by the surgeon or pulmonologist. We demonstrate this $3 \mathrm{D}$ software in patients with advanced emphysema, who were scheduled for LVRS, assessed outcome and we demonstrate typical cases to illustrate the role of morphology in patient selection and planning surgery.

\section{Methods}

Software and computer tomography

A real-time 3D image analysis software system (Synapse Vincent, Fuji Film Co., Ltd., Tokyo, Japan) was used pre- and 3 months post-operatively in six consecutive patients with severe emphysema scheduled for LVRS. These patients were already scheduled for an institutional LVRS course for teams of surgeons, pulmonologists and radiologists over 2 days. CT scan slices with $2-\mathrm{mm}$ slice thickness were analysed. The pre-operative CT was performed within 3 months before the operation. The post-operative CT was scheduled 3 months after LVRS.

The software system collects the soft-tissue sequences from the institutional picture archiving and communication system and the user can initiate an automatic process to create the densitometry of the lung.

User-dependent, low attenuation areas (LAAs) can be defined by setting the Hounsfield units. For this trial, a range between -951 and $-1000 \mathrm{HU}$ was defined as severe emphysema and colour-coded with red [7]. Coloured images were demonstrated and used for patient selection at the interdisciplinary emphysema board and for planning surgery. At least two surgeons experienced in LVRS, including the last author of this study, interpreted the pictures for planning the procedure.

\section{Lung function and follow-up}

Clinical examination, CT scans and pulmonary lung function tests (PFTs) consisting of spirometry, body plethysmography and single-breath CO diffusion were performed pre-operatively and 3 months after surgery.

\section{Inclusion criteria for LVRS}

Symptomatic patients with advanced emphysema and a dyspnoea score of MRC 3 and 4 were considered and those with a residual volume (RV)/TLC ratio $>0.58$ were included and patients with a diffusing capacity of the lung for CO $\left(D_{\mathrm{LCO}}\right) \leqslant 20 \%$ were excluded, except in patients with an obvious target zone of major emphysematous destruction on CT. There are no strict limits regarding $\mathrm{FEV}_{1}$, but most LVRS patients show $<45 \%$. All types of emphysema morphology are considered for LVRS, but finally selected in a synopsis based on lung function, gas exchange and emphysema morphology. All patients are discussed 


\section{TABLE 1 Pre- and post-operative lung function values of all six patients}

\begin{tabular}{llll} 
& Pre & Post & p-value \\
\hline FEV $_{\mathbf{1}} \mathbf{~ m L}$ & $665(525-895)$ & $935(802-1387)$ & 0.028 \\
FEV $_{\mathbf{1}} \%$ & $23(21.5-29.3)$ & $39(34.5-47.5)$ & 0.028 \\
TLC mL & $8105(6265-10222)$ & $6220(5052-7107)$ & 0.116 \\
TLC $\%$ & $129(113-157)$ & 0.916 \\
RV $\mathbf{~ L L}$ & $144(108-164)$ & $2405(2080-2955)$ & 0.498 \\
RV \% & $2375(2035-3065)$ & $168(149-252)$ & 0.028 \\
RV/TLC & $279(210-288)$ & $51(47-68)$ & 0.027 \\
$\boldsymbol{D}_{\text {LCo } \%}$ & $73(68.5-78.5)$ & $34(25-49)$ & 0.042
\end{tabular}

Data are presented as median (range). $\mathrm{FEV}_{1}$ : forced expiratory volume in $1 \mathrm{~s}$; TLC: total lung capacity; RV: residual volume; $D_{\mathrm{LCO}}$ : diffusing capacity of the lung for carbon monoxide.

at our interdisciplinary emphysema treatment board with thoracic surgeons and pulmonologists participating, while the CT scans are additionally analysed by dedicated chest radiologists. Hyperinflation is also radiologically evaluated using signs such as flattened diaphragm and funnel chest. $\mathrm{FEV}_{1}$ and diffusion capacity values below $20 \%$ predicted are only accepted in cases of heterogeneous emphysema [13] where the target area of resection is (as suggested from perfusion scan and CT scan) obviously functionless lung tissue. Concerning homogeneous emphysema, patients with severe hyperinflation and a $D_{\text {LCO }}>20 \%$ predicted are also considered as possible surgical candidates.

\section{Pre-operative cardiac assessment}

At our institution, pre-operative myocardial single-photon emission CT scans are performed to assess cardiac ischaemia in high-risk patients. These include patients with a history suggestive of cardiac disease (high-risk factor profile or angina) and/or the inability to climb more than one flight of stairs. All patients underwent screening Doppler transthoracic echocardiography. Systolic pulmonary artery pressure (sPAP) $>35 \mathrm{mmHg}$ is a strict contraindication for LVRS in patients with homogeneous emphysema. sPAP up to $45 \mathrm{mmHg}$ is tolerated for those with heterogeneous emphysema and severe hyperinflation.

\section{Surgery}

In general, a bilateral LVRS is intended and a unilateral approach was selected for patients with unilateral dominated disease, or when severe adhesions were found during surgery on the first side. A supine position with both arms raised was used for upper-lobe-predominant emphysema and for purely homogeneous emphysema. Bilateral LVRS for combined upper and lower-lobe-predominant disease or unilateral LVRS was performed in a lateral decubitus position. The position was changed under the same anaesthesia after termination of the first side. General anaesthesia was conducted with a left-sided double lumen tube [14]. We performed video-assisted thoracic surgery (VATS) with three ports. Volume reduction was performed with stapling devices aiming to resect the most destroyed parts to remodel the lungs to the predicted TLC. One chest tube per side was introduced. The chest tube was connected with a low-pressure suction water system $\left(-5 \mathrm{~cm} \mathrm{H}_{2} \mathrm{O}\right)$ and the patient was extubated via laryngeal mask in the operation room. Post-operatively, the patient was transferred to the intermediate care station until the next morning before returning to the ward. The intensive care unit is rarely required.

\section{TABLE 2 Perioperative course}

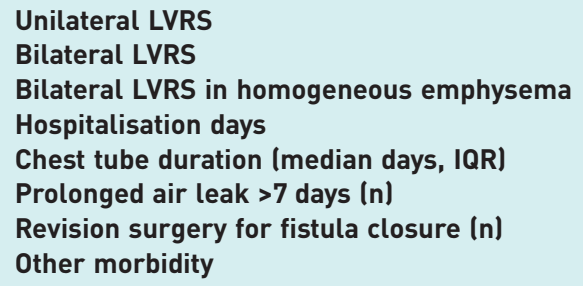

Data are presented as $\mathrm{n}$ or median (interquartile range). LVRS: lung volume reduction surgery; IQR: interquartile range. 


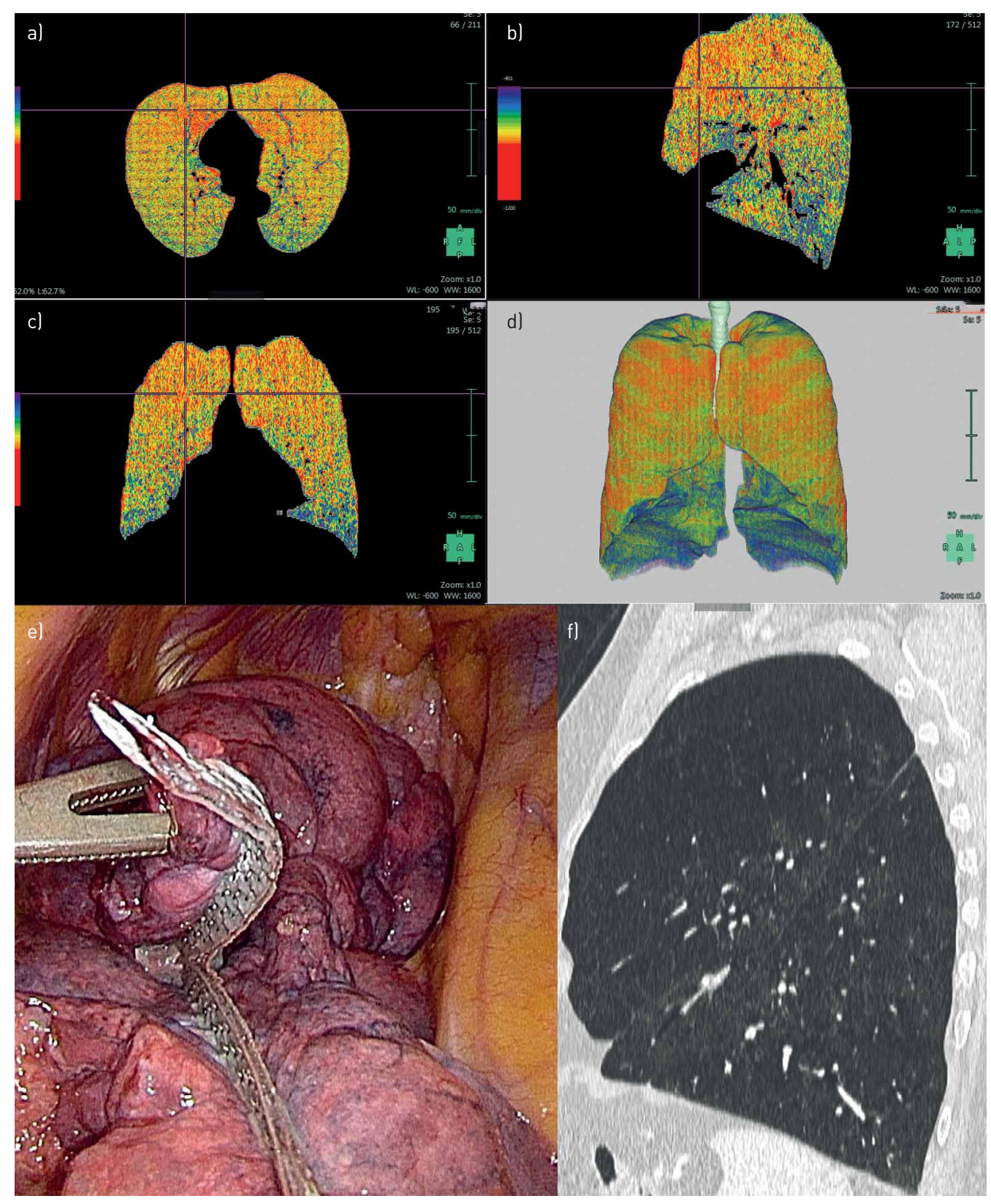

FIGURE 1 Patient 1. a-d) Densitometry: bilateral upper-lobe-predominant emphysema. e) Intra-operative: resection at the upper lobe on the right side. f) Computed tomography: heterogeneous emphysema at the left upper lobe.

\section{Statistics}

Descriptive statistics were used to summarise patients' characteristics. Continuous variables were reported as median and range and compared between the two groups using two-sample independent t-tests or a Mann-Whitney U-test (non-normal data). Comparisons of pre- and post-operative values originating from the same patient were compared using the Wilcoxon signed-rank test (non-normal data). All reported p-values are two-sided, with a significance level of 0.05 . SPSS version 22 (IBM SPSS Statistics for Windows, Armonk, NY, USA: IBM Corp., 2013) was used for data analysis.

\section{Ethical approval}

The institutional review board approved this study (KEK no. 2016-00716).

\section{Results}

All patients had bilateral emphysema, almost equally distributed on both sides. Three patients had an upper-lobe-predominant type of emphysema. Two patients had predominant areas of destruction 


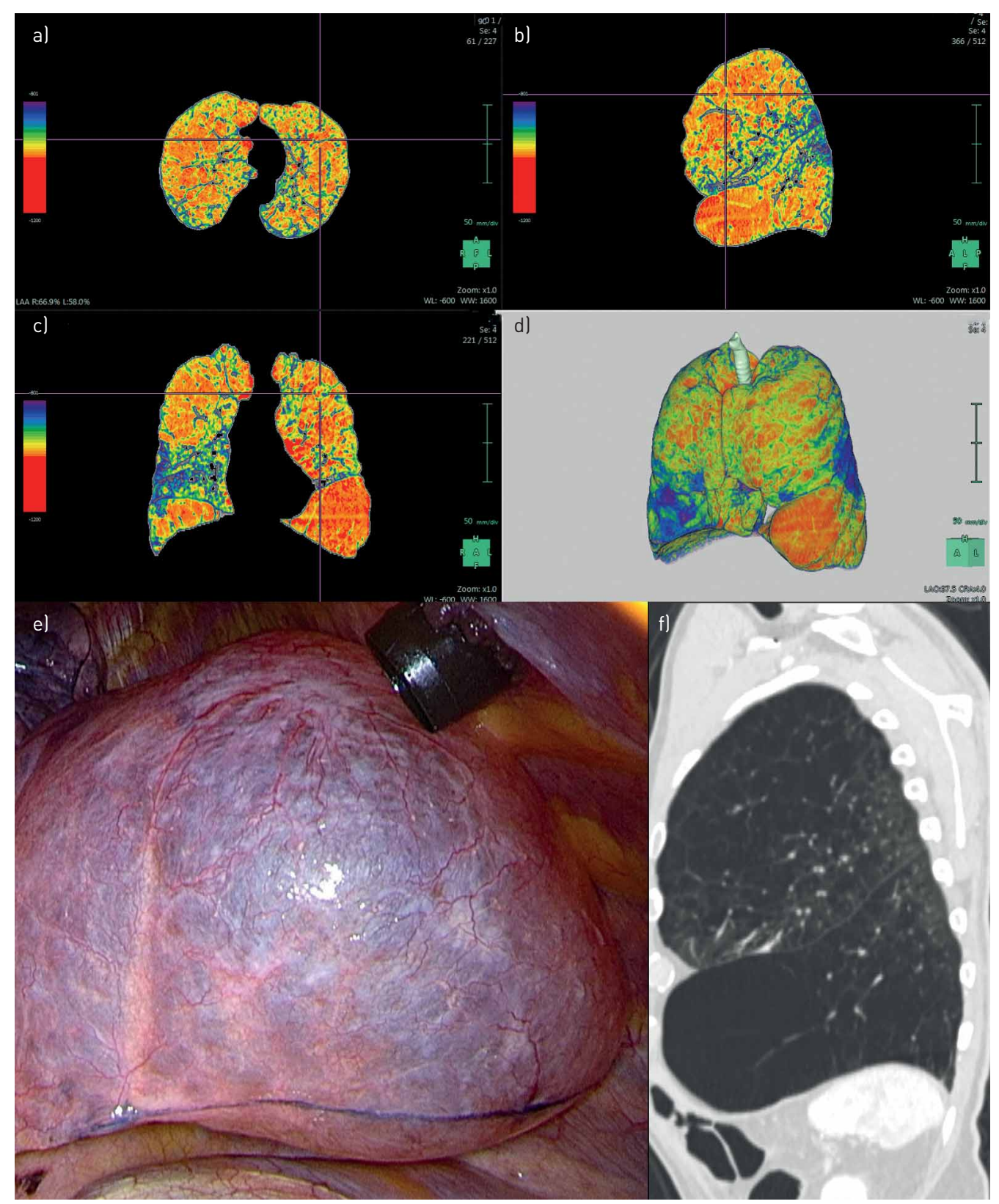

FIGURE 2 Patient 2. a-d) Densitometry: bilateral upper- and lower-lobe-predominant emphysema. e) Intra-operative: bullous-like emphysema at the left lower lobe. f) Computed tomography: heterogeneous emphysema at the left upper lobe and at the anterior part of the lower lobe.

combined in the upper and lower lobe and one patient had a purely homogeneous emphysema type. Table 1 shows the pre- and post-operative pulmonary function values. Post-operative $\mathrm{FEV}_{1} \%$ predicted showed an increase by $70 \%$ (range 30-120\%) compared with baseline values. Hyperinflation (RV/TLC) was reduced by $30 \%$ (range $5-32 \%$ ). $D_{\text {LCO }}$ increased by $31 \%$ (range $0-42 \%$ ).

Five patients had bilateral LVRS (including the one with homogeneous emphysema) and one patient had an extended adhesiolysis on the right side and after placing the camera port on the left side, the operation was terminated prematurely because of evidence of massive adhesions. All operations were performed by VATS. Table 2 shows the patient's post-operative course. Figures 1-6 demonstrate each patient's CT morphology, densitometry and intra-operative view. Table 3 shows the $\mathrm{FEV}_{1} \%$ predicted and percentage of LAA side by side pre- and post-operatively for each individual patient. Regarding $\mathrm{FEV}_{1}$, medians of all patients (pre- versus post-operatively) were calculated. All patients with heterogeneous emphysema showed a clear reduction in \% LAA post-operatively compared to pre-operatively, medians were calculated, excluding the patient with homogeneous disease. The latter showed no reduction in \% LAA, in accordance 

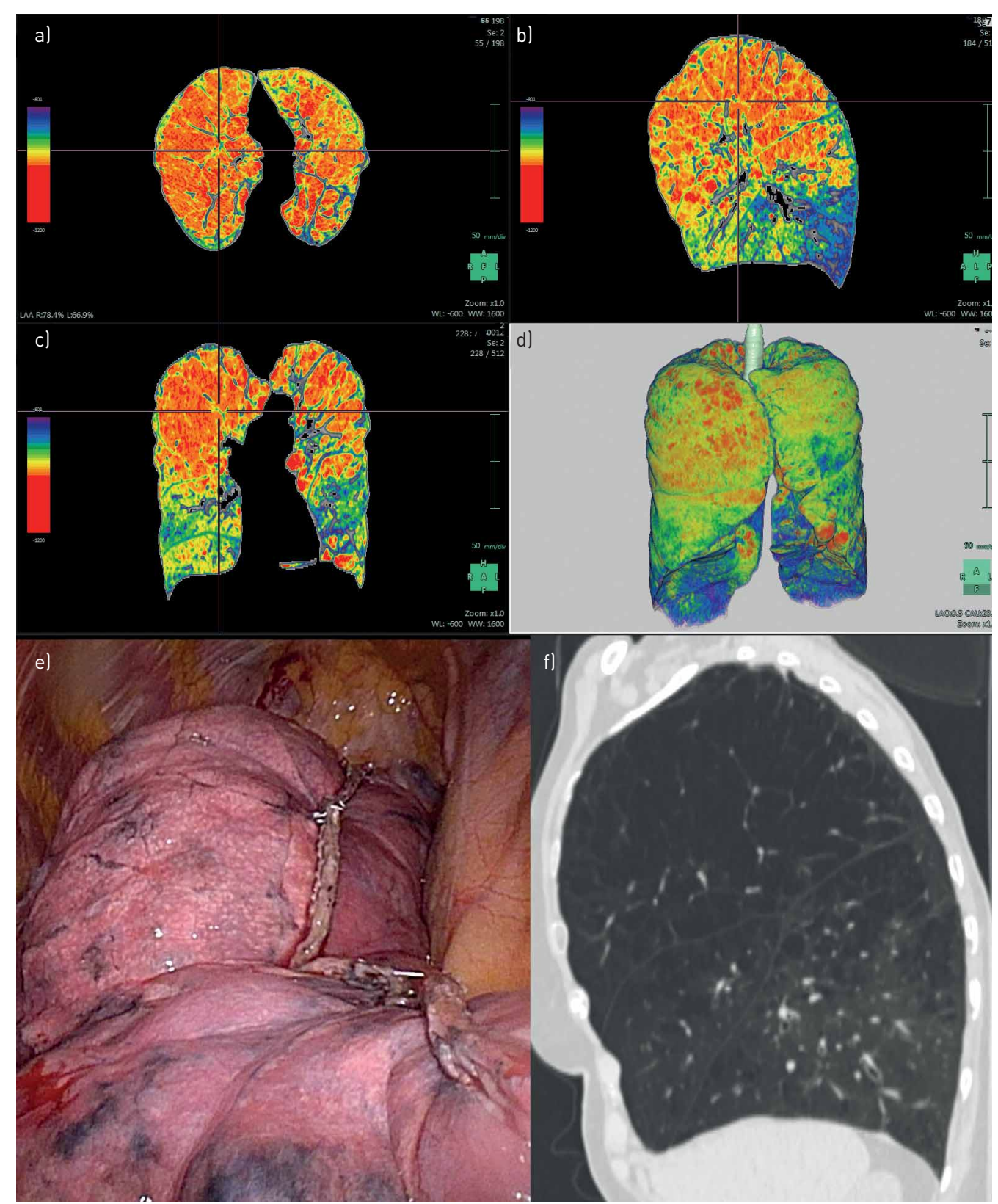

FIGURE 3 Patient 3. a-d) Densitometry: bilateral upper-lobe-predominant emphysema, more prominent on the right side. e) Intra-operative: lung volume reduction surgery at the upper lobe on the right side. f) Computed tomography: heterogeneous emphysema at the right upper lobe.

with the even distribution of emphysema. Patient number three also showed no left \% LAA reduction, as the operation was unilateral.

\section{Discussion}

LVRS is aimed to remodel the lungs to reduce hyperinflation in a personalised way by resecting the most functionless tissue. In order to find the target area and to define the planned volume for resection, traditionally each individual slice of the CT scan is studied by the surgeon and a plan is constructed by imagination of a 3D lung.

In this study, we demonstrate a software applicable to chest CT scans, which enables LAAs to be highlighted in two dimensions and in 3D, which can be more easily interpreted and converted into a resection plan. Furthermore, it might help also with patient selection, as a synopsis has to be made considering emphysema morphology and PFTs. Showing examples of six consecutive LVRS patients with different morphologies, we demonstrate the role of colour-coded densitometry based on CT for operative 


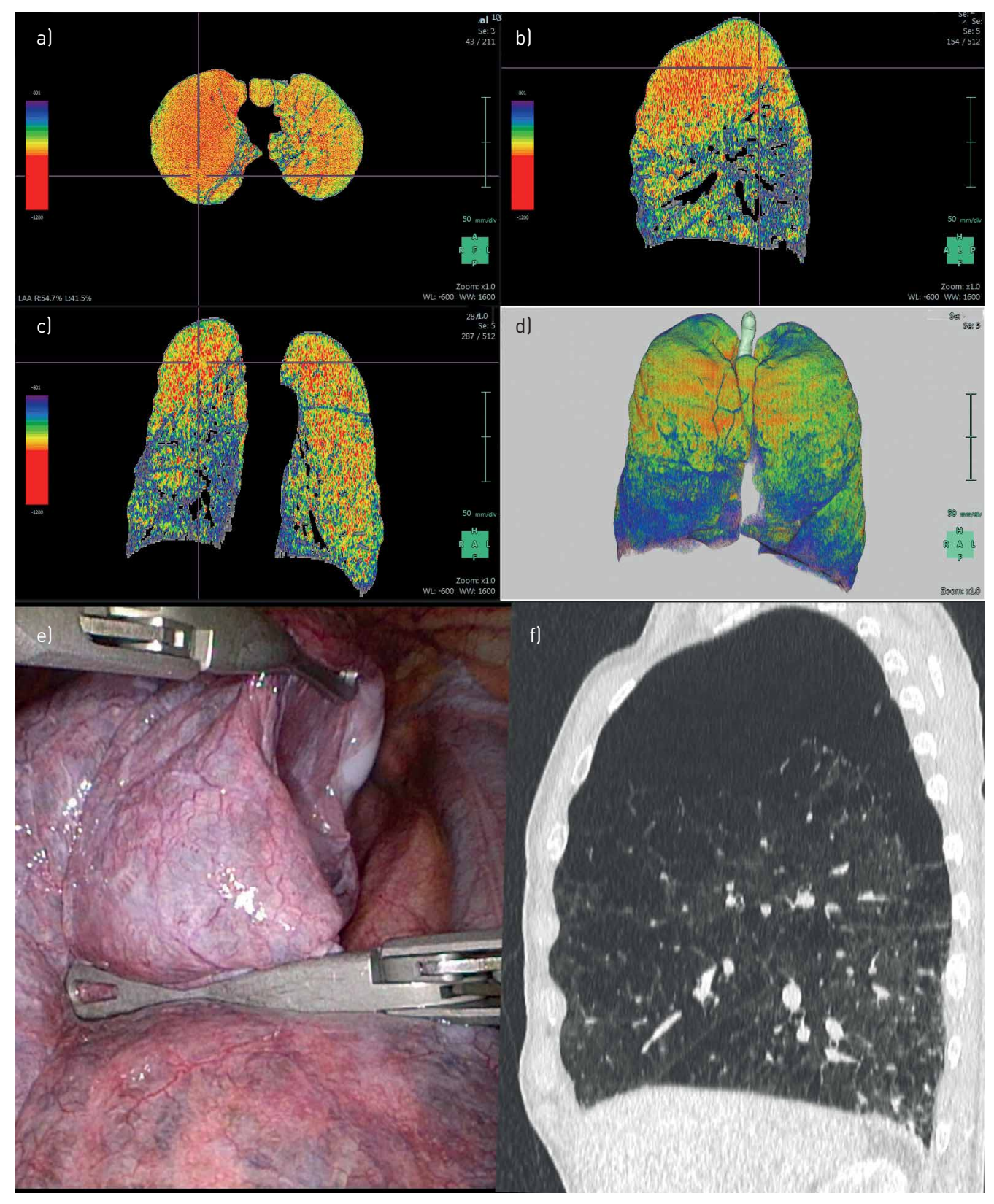

FIGURE 4 Patient 4. a-d) Densitometry: bilateral upper-lobe-predominant emphysema. e) Intra-operative: simulating resection at the upper lobe on the right side. f) Computed tomography: heterogeneous emphysema at the right upper lobe.

planning and correlate it with PFTs and plethysmography, before surgery and 3 months post-operatively. PFTs showed a clear improvement and the densitometries showed a decrease, assessed by areas of low attenuation. LVRS on the right side showed a LAA reduction from $45.9 \%$ to $39.8 \%$, suggesting that the important target areas were successfully resected as planned. The patient with homogeneous emphysema morphology was not included in the analysis regarding reduction of \% LAA (table 3) for obvious reasons, because there is no LAA reduction possible due to an equal emphysema density distribution throughout the lungs. The left side showed a clear reduction from $54.3 \%$ to $44.6 \%$. Four patients were available, as one LVRS in heterogeneous emphysema was performed unilaterally on the right side only due to unexpected severe adhesions and the decision was made not to expose the patient to the risk of a difficult post-operative course. Anyway, measuring lung volumes on the left side has been reported to be more challenging. MARTINI et al. [15] described difficulties in obtaining reliable and repeatable measurements on the left side during dynamic magnetic resonance imaging of the lungs before and after LVRS. The heart might possibly limit lung parenchymal expansion. Hightower et al. [16] stated that measurements of the 

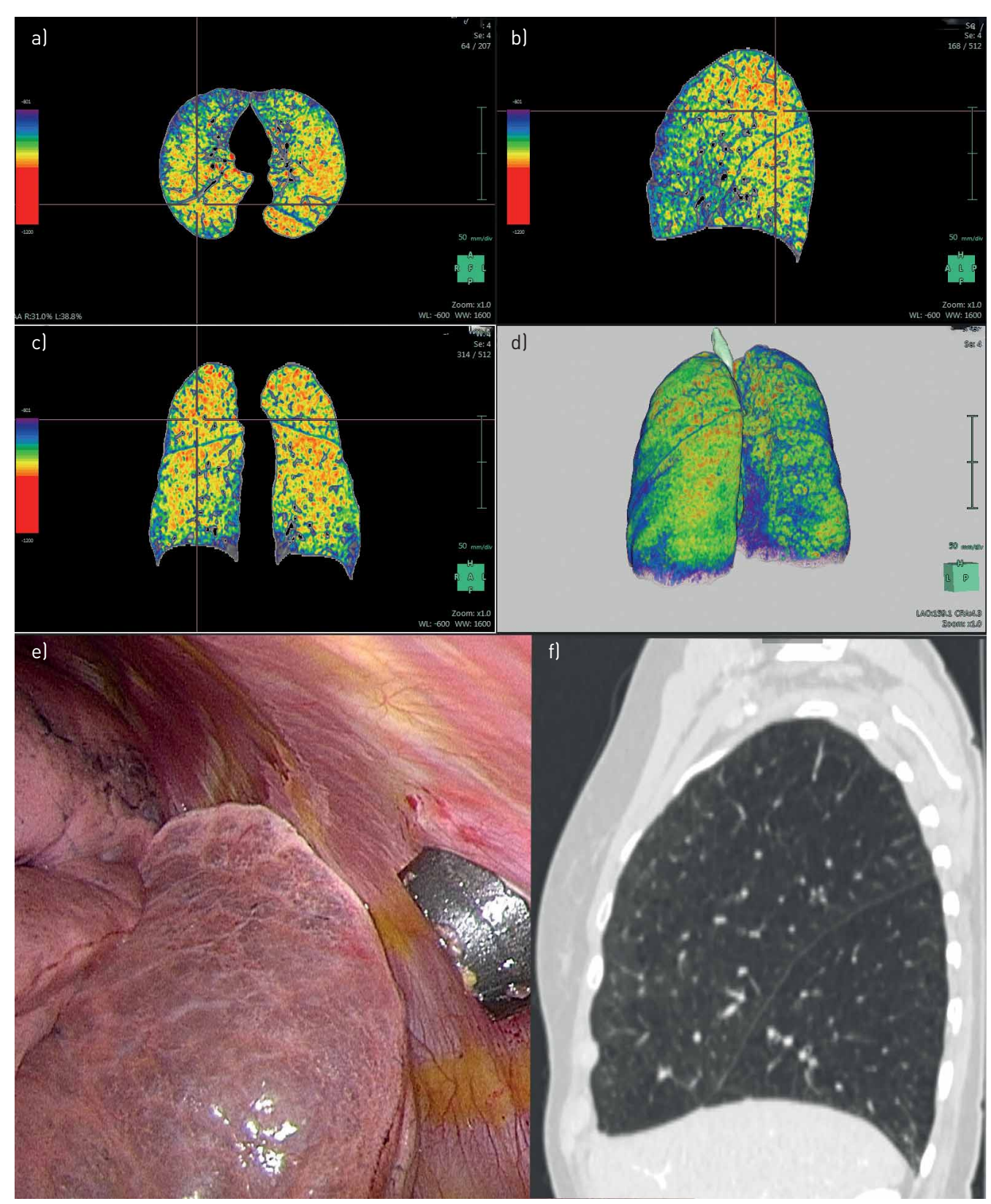

FIGURE 5 Patient 5. a-d) Densitometry: bilateral upper-lobe and apical lower-lobe-predominant emphysema. e) Intra-operative: emphysema at apical left lower lobe. f) Computed tomography: heterogeneous emphysema at the left upper and apical lower lobe.

right lung seem to be more influenced by typical emphysematous changes such as flattened (hemi) diaphragm and increased lung height [16]. Nevertheless, our results regarding post-operative decrease of LAA suggest the reliability of the utilised software, despite the low number of patients. Applicability of densitometry for emphysema patients has recently been shown by our group [9]. Colour-coded images improved inter-reader agreement of characterising emphysema distribution and providing resection recommendations. Three readers assessed visually the amount and distribution patterns of emphysema on axial, multiplanar and densitometry CT images. They used the Goddard system and our own LVRS-oriented classification system (heterogeneous versus intermediately heterogeneous versus homogeneous, [17]). The results showed a substantial inter-rater agreement for emphysema distribution for the densitometry (Fleiss' $\kappa=0.70)$, as well as in comparison to axial and multiplanar images $(\mathrm{p}<0.001)$. In 2007, Sтоцк et al. [18] explored changes in the extent of emphysema compared with changes in lung function after LVRS. They used CT densitometry in 30 LVRS patients and found a correlation between decreasing $\mathrm{RV}$, increasing diffusion capacity and increasing lung density. Further patient series might be 


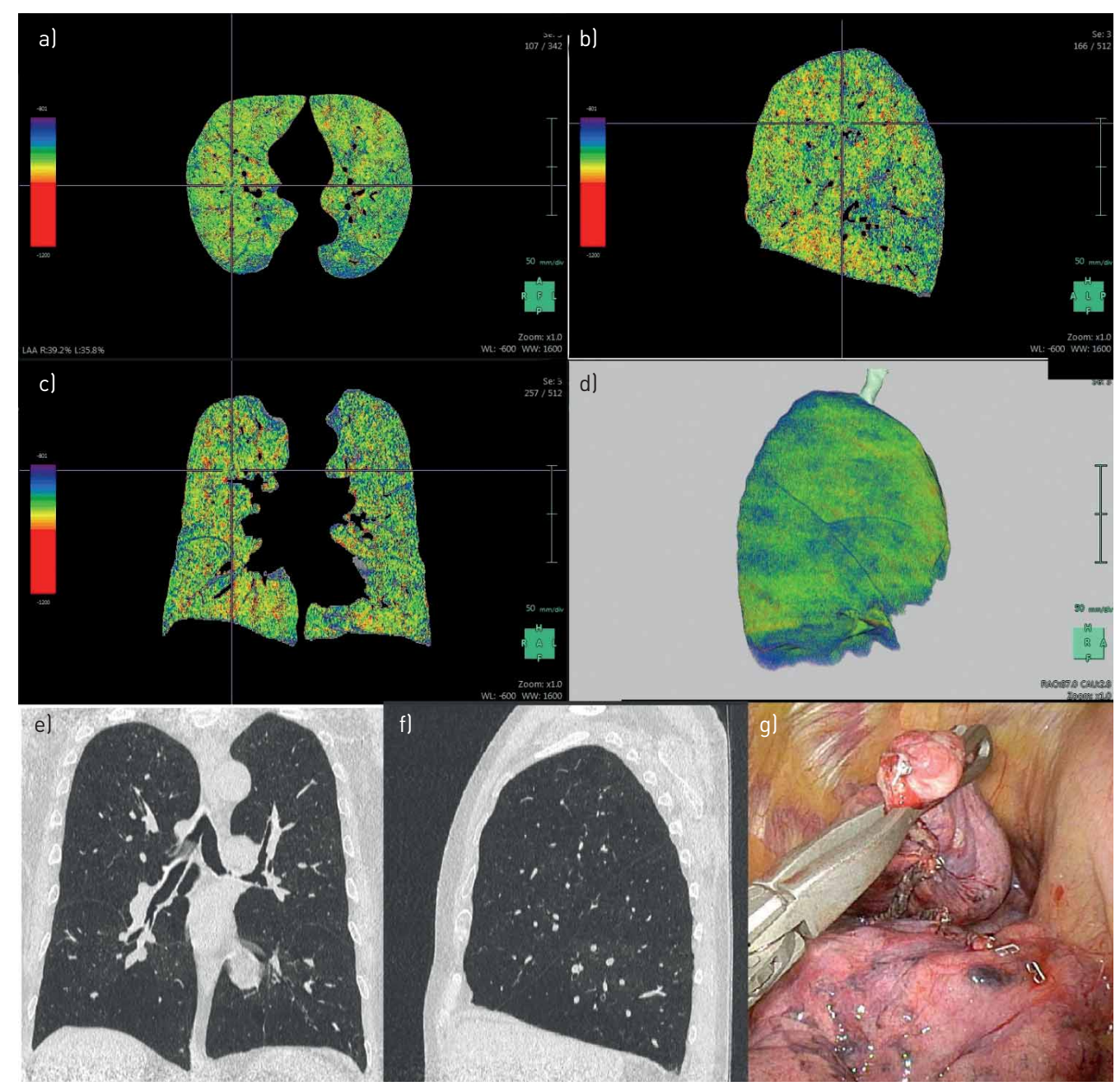

FIGURE 6 Patient 6. a-d) Densitometry: purely homogeneous emphysema. e, f) Computed tomography: homogeneous emphysema. g) Intra-operative: apical resection at the right upper lobe.

necessary to further confirm a statistically significant correlation between decreased LAA and improvement of pulmonary function after LVRS. Once more, our results in this small study show marked improvements of PFTs after LVRS (table 1), demonstrating that the right target areas were resected and most likely the right volume. The latter is impossible to define and study. We assume and intend to achieve the re-establishment of a TLC as predicted.

Another advantage of this software is its user friendliness. It can be applied by the clinician for patient selection and for planning surgery without the guidance of a radiologist. Of course, we will still include a dedicated radiologist in the patient evaluation, but planning surgery has to be done by the surgeon. Whereas the obvious upper-lobe-predominant type of emphysema gives few problems in identifying the target area (figures 1-4), the identification of possible targets in other locations is much more challenging. As we have demonstrated for many years that well-selected patients with intermediate heterogeneous and even homogenous emphysema can also profit from LVRS [2,17] the correct assessment of morphology is essential and improved using 3D imaging. The example in figure 5 shows a relevant target zone in the upper part of the left lower lobe, which is well visible on 3D imaging and densitometry and further confirmed intra-operatively. The CT scan alone did not clearly allow the identification of this zone with very low attenuation. In the case of homogeneous emphysema, the software might further help to confirm pure homogeneity.

Endobronchial procedures such as valves, coils and vapour are also intended to selectively eliminate the most destroyed emphysematous lung areas [19]. Valves eliminate an entire lobe by creating a total atelectasis [20]. There, 3D CT might be of value to assess the morphology of a lobe and calculate the amount of tissue with some remaining function that will be eliminated. Vapour is intended to selectively shrink an area of the most destroyed tissue and there the described technology might also be of value. 


\section{TABLE 3 Pre- and post-operative values of FEV 1 and \% LAA (right and left side) of each patient}

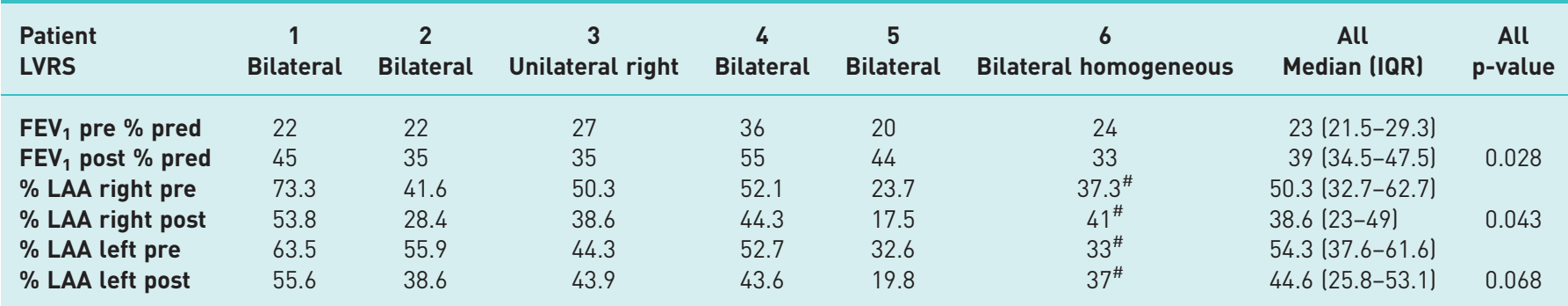

$\mathrm{FEV}_{1}$ : forced expiratory volume in $1 \mathrm{~s}$; LAA: low attenuation area; LVRS: lung volume reduction surgery; IQR: interquartile range. ${ }^{\#}$ : not included for analysis of median and $p$-values.

To conclude, the application of the 3D imaging software allows the selection and identification of target regions for resection and the planning of surgery more precisely. It might help additionally in the future to select the best technique (endobronchial or surgical) for an individual patient.

Conflict of interest: C. Caviezel has nothing to disclose. T. Froehlich has nothing to disclose. D. Schneiter has nothing to disclose. U. Muehlematter has nothing to disclose. T. Frauenfelder has nothing to disclose. L-C. Guglielmetti has nothing to disclose. I. Opitz has nothing to disclose. W. Weder reports personal fees for teaching support from Medtronic outside the submitted work.

\section{References}

1 Darwiche K, Aigner C. Clinical management of lung volume reduction in end stage emphysema patients. $J$ Thorac Dis 2018; 10: Suppl. 23, S2732-S2737.

2 Caviezel C, Schneiter D, Opitz I, et al. Lung volume reduction surgery beyond the NETT selection criteria. $J$ Thorac Dis 2018; 10: Suppl. 23, S2748-S2753.

3 Cassart M, Hamacher J, Verbandt Y, et al. Effects of lung volume reduction surgery for emphysema on diaphragm dimensions and configuration. Am J Respir Crit Care Med 2001; 163: 1171-1175.

4 Lim E, Sousa I, Shah PL, et al. Lung volume reduction surgery: reinterpreted with longitudinal data analyses methodology. Ann Thorac Surg 2019; 109: 1496-1501.

5 National Emphysema Treatment Trial Research Group, Fishman A, Fessler H, et al. Patients at high risk of death after lung-volume-reduction surgery. N Engl J Med 2001; 345: 1075-1083.

6 Fishman A, Martinez F, Naunheim K, et al. A randomized trial comparing lung-volume-reduction surgery with medical therapy for severe emphysema. N Engl J Med 2003; 348: 2059-2073.

7 Bankier AA, De Maertelaer V, Keyzer C, et al. Pulmonary emphysema: subjective visual grading versus objective quantification with macroscopic morphometry and thin-section CT densitometry. Radiology 1999; 211: 851-858.

8 Hersh CP, Washko GR, Jacobson FL, et al. Interobserver variability in the determination of upper lobe-predominant emphysema. Chest 2007; 131: 424-431.

9 Muehlematter UJ, Caviezel C, Martini K, et al. Applicability of color-coded computed tomography images in lung volume reduction surgery planning. J Thorac Dis 2019; 11: 766-776.

10 Ikeda N, Yoshimura A, Hagiwara M, et al. Three-dimensional computed tomography lung modeling is useful in simulation and navigation of lung cancer surgery. Ann Thorac Cardiovasc Surg 2013; 19: 1-5.

11 Hagiwara M, Shimada Y, Kato Y, et al. High-quality 3-dimensional image simulation for pulmonary lobectomy and segmentectomy: results of preoperative assessment of pulmonary vessels and short-term surgical outcomes in consecutive patients undergoing video-assisted thoracic surgery. Eur J Cardiothorac Surg 2014; 46: e120-e126.

12 Chen-Yoshikawa TF, Date H. Update on three-dimensional image reconstruction for preoperative simulation in thoracic surgery. J Thorac Dis 2016; 8: Suppl. 3, S295-S301.

13 Caviezel C, Schaffter N, Schneiter D, et al. Outcome after lung volume reduction surgery in patients with severely impaired diffusion capacity. Ann Thorac Surg 2018; 105: 379-385.

14 Grande B, Loop T. Anaesthesia management for bronchoscopic and surgical lung volume reduction. J Thorac Dis 2018; 10: Suppl. 23, S2738-S2743.

15 Martini K, Caviezel C, Schneiter D, et al. Dynamic magnetic resonance imaging as an outcome predictor for lung-volume reduction surgery in patients with severe emphysema. Eur J Cardiothorac Surg 2018; 105: 379-385.

16 Hightower JS, Amadi C, Den E, et al. Back to the future: sagittal CT in the evaluation of COPD. Eur Radiol 2016; 26: $2730-2739$

17 Weder W, Thurnheer R, Stammberger U, et al. Radiologic emphysema morphology is associated with outcome after surgical lung volume reduction. Ann Thorac Surg 1997; 64: 313-319.

18 Stolk J, Versteegh MI, Montenij LJ, et al. Densitometry for assessment of effect of lung volume reduction surgery for emphysema. Eur Respir J 2007; 29: 1138-1143.

19 van Geffen WH, Slebos DJ, Herth FJ, et al. Surgical and endoscopic interventions that reduce lung volume for emphysema: a systemic review and meta-analysis. Lancet Respir Med 2019; 7: 313-324.

20 Criner GJ, Sue R, Wright S, et al. A multicenter randomized controlled trial of zephyr endobronchial valve treatment in heterogeneous emphysema (LIBERATE). Am J Respir Crit Care Med 2018; 198: 1151-1164. 